\title{
The modeling and simulation of intelligence information process of command and control system based on IDEF and Petri nets
}

\author{
Xianglin $\mathrm{Tan}^{1,}$, Fuqi $\mathrm{Qu}^{1}$, Jianhua $\mathrm{Tu}^{1}$ \\ ${ }^{1}$ Wuhan Mechanical College, Wuhan, HuBei, 430075, China \\ atxl19861017@163.com
}

\begin{abstract}
Keywords: IDEF, Petri nets, Intelligence information process, Modeling \& simulation
Abstract. Intelligence activities are the basic activities of operational command, which have features of asynchronous and concurrent, that is difficult to use traditional mathematical tools to analyze. On the basis of analysis of digital division intelligence information process of activities, combined with the IDEF methodology and intelligence information process model established by Petri nets, evidenced by the model simulation run results: the model can more really describe the intelligence activities process and can effectively analyze the rationality and feasibility of command work organization.
\end{abstract}

\section{Introduction}

Intelligence activities is one of the basic activities of battle command, which is the premise of decision, control and coordinate activities, and its purpose is to timely, accurate and comprehensive to grasp of all kinds of information related to the battle and battle command, which provide intelligence information security for battle command [1,2].Under the current conditions of informatization, relying on command and communication system intelligence acquisition and analysis process is driven by a discrete event distributed real-time multi-tasking process which has asynchronous, concurrent, etc, it is difficult to use them to build a single mathematical tools for modeling.

In this paper, we study for the US digital Armored Division intelligence information flow, combined with IDEF methods and Petri nets from different levels to decomposition and description of intelligence information flow, and to construct the corresponding simulation model to analyze the impact on making decision under different information intensity and different processing conditions.

\section{The flow of digital armored division intelligence information activities}

The intelligence information of digital armored division based communications network as a support and the intelligence sections as the core, with the combat reconnaissance unit entities intelligence source, relying on alleged communications system builders "intelligence information sharing network", "unified planning, decentralized collection, centralized processing, sequential distribution, high degree of sharing" of the integration of information protection mechanism. From the perspective of the process of intelligence information, the intelligence information flow of digital armored division mainly comes by information acquisition, information processing and information distribution three processes. The basic information process of the intelligence activities is shown in figure 1. 


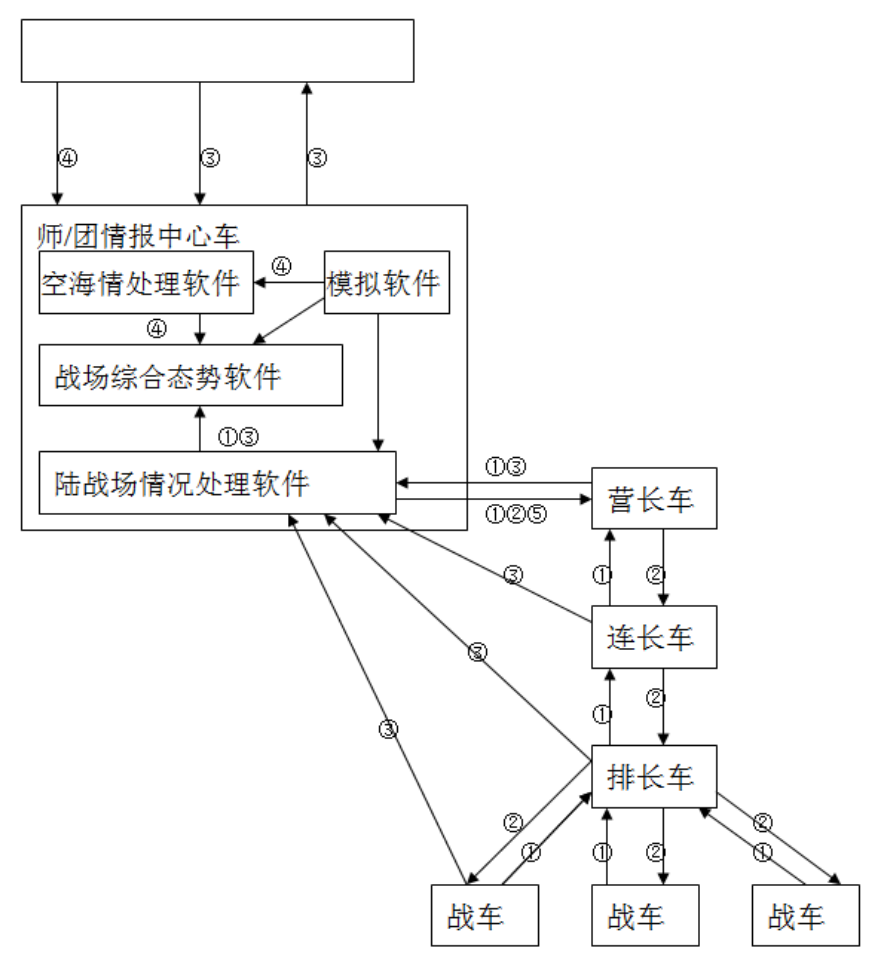

Figure 1 Intelligence activity information process

\section{Intelligence information process modeling based on IDEF}

IDEF method has the advantage which can decompose step by step from the view point of system, objective system are internal activity, contact and the object and the relationship between the module and can clearly said, information input and output relationship between each command node relation model made by as close as possible to the actual process[3,4]. The following is the modeling process description:

\subsection{Establish internal and external relationship figure: A - 0 figure}

Establish internal and external relations A - 0 figure, determine the internal and external relationship of the whole system, determine the boundary of the system and the external connection, form the foundation of the decomposition. Modeling is usually the first step in building internal and external relationship figure: A - 0 figure. Draw a single box, box indicates the name of the function process and the name can summarize the entire described contents. And then the arrow out of the box indicates the data interface system environment, as shown in figure 2.

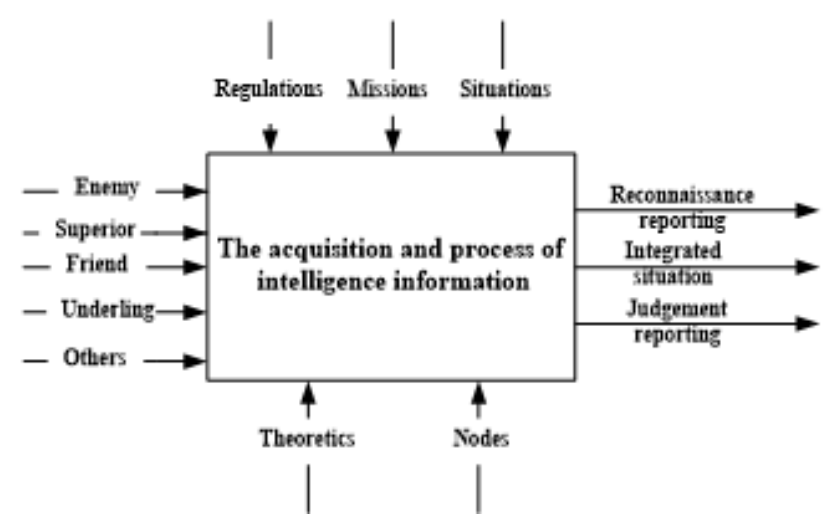

Figure 2 A - 0 figure of acquisition and process of intelligence information

\subsection{Determine the function and instruction set}

After the establishment of internal and external relations A-0 diagram, the second step to determine the function and instruction set, depending on the different uses of command and control process, then determine the different function and instruction set, and marks the different functions 
and information property. For example, this information receiving box includes the situation of the enemy, the superior conditions, friendly intelligence support and other information, as shown in figure 3.
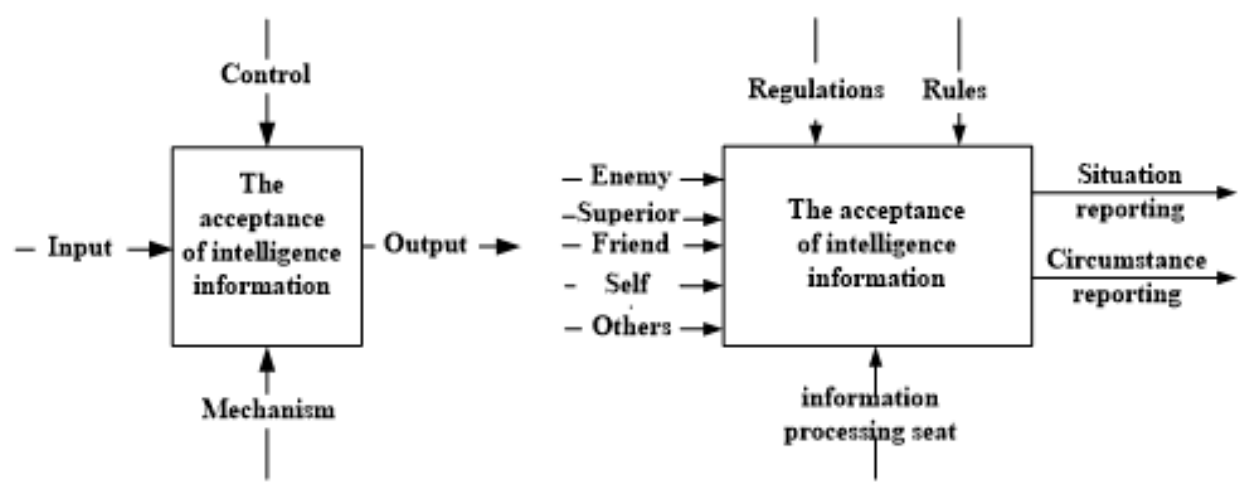

Figure 3 Receive box border and functions and commands collection of intelligence information

\subsection{Draw the top chart and the sub-graph}

According to the determined set of function, we design top activity figure which decompose A-0 figure into the required parts, this is actually the top chart of the system model. It describes the main features of the system from the structure. A-0 structure diagram which clearly shows the meaning of A-0 box to be described, the lower-level graphics than A-0 that illustrates the contents of each box to be described.

Acquisition and processing of intelligence information can be broken down into five main sections: information receiving, information integration, information interfusion, and information reporting and information distribution, draw the top as shown in figure 4.

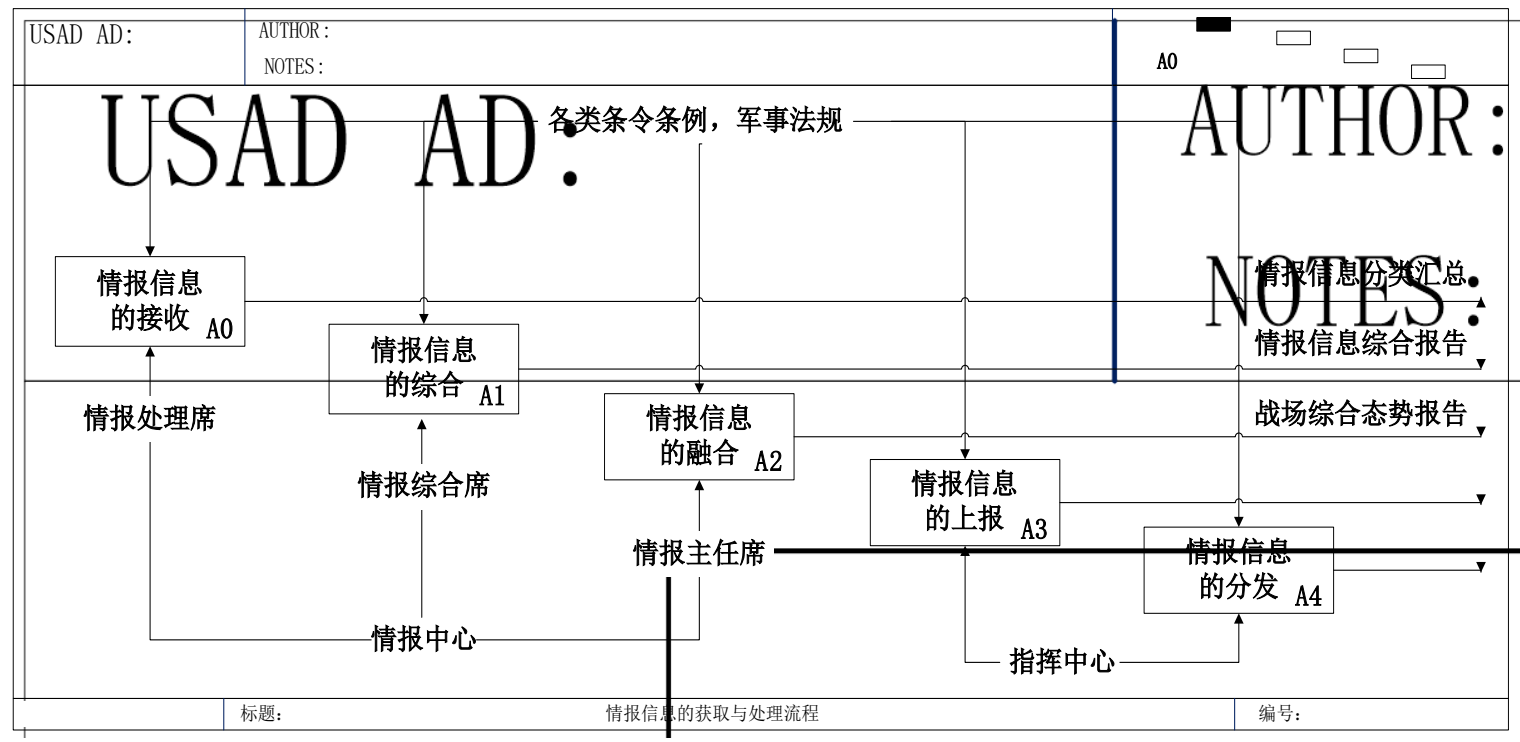

Figure 4 Top chart of acquisition and processing of intelligence information

\subsection{Add relevant text}

Text explain as a short and precise depiction to the graphs which add important information that the figures cannot express, while explain and illustrate the vocabularies of the figures.

\section{The simulation of intelligence information process based on Petri nets}

Because of IDEF cannot describe dynamic characteristic of the system, and then we introduce Petri nets. Petri nets are particularly easy to describe system process or the relationships of sequence, concurrency, conflict and synchronous of the components [5, 6]. In this section, we will apply simulation software of object Petri net to support environment OPMSE based on IDEF model. The modeling process can be divided into: 


\subsection{Edit Petri nets libraries}

The intelligence information processing can be divided into five parts: intelligence information decomposition, acquisition, fusion, reporting and distribution. Then we describe working process of intelligence tasks according to intelligence tasks decomposition, and its OPDL description is shown in figure5.

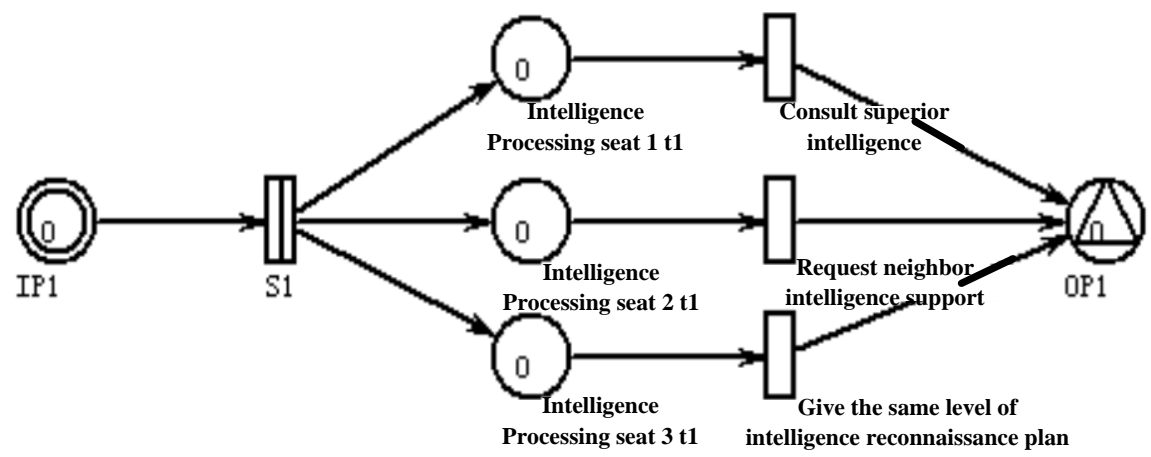

Figure 5 The OPDL description of intelligence tasks decomposition

Transfers of the figure are described as follows: Request superior intelligence: verify intelligence information from higher intelligence center; Request neighbor intelligence support: send the relevant intelligence information support to the neighbor; Detach intelligence reconnaissance plan to the same level: assign reconnaissance scheduled tasks to their respective reconnaissance detachments. The meaning of switches of the figure is illustrated as follows: S1: according to seat for intelligence tasks distribution. The meanings of ports in the figure are illustrated: OP1: output port, which is used to send intelligence task information to superiors, neighbors and the same level of intelligence detachments; IP1: input port, which is used to receive intelligence task indication information from the command center.

\subsection{Generate the model file.}

We select an edited model through the management window, then "Binding", we generate an .ope file, and this is the model file.

\subsection{Simulation running}

Before the simulation of the model, we should configure parameter in conjunction with initialization function and event handler functions, and then complete simulation environment and model initialization. Then we begin to simulate by using the "execution" or "single step" command on the toolbar. For intelligence information flow, we need to mathematical quantify the input intelligence which tries to make the data size and process match actuality, so the simulation results are able to verify the actual system operating conditions and make the adjustment and optimization more proof. Running result of OPMSE model simulation is shown in figure 6.

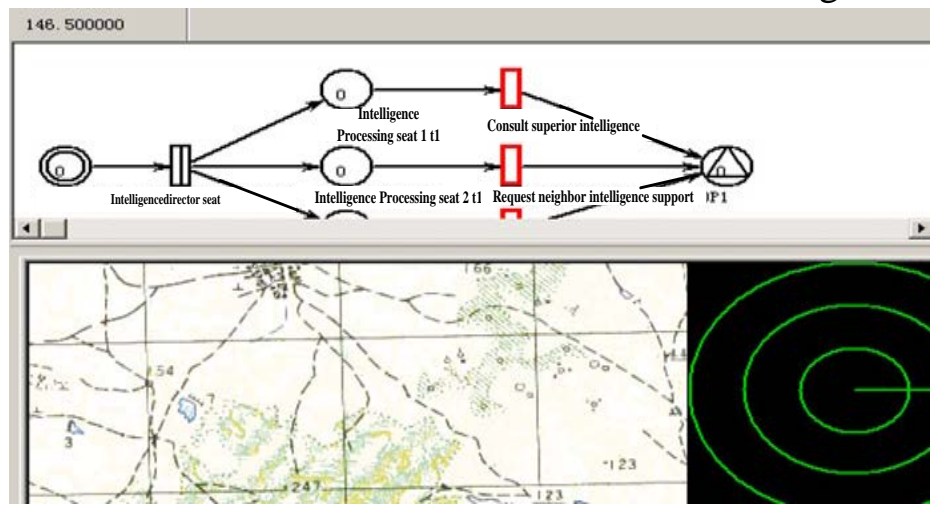

Figure6 Running result of OPMSE model simulation

In this simulation environment, we modify the delay time of intelligence comprehensive analysis that we can obtain the influence to decision-making under different information intensity and different processing conditions. In this article, we set delay time to random numbers of a certain 
distribution, through several simulations to get the relationship between effective reporting battlefield situation probabilities distributing and the amount of information and processing flow, as shown in Figure7.

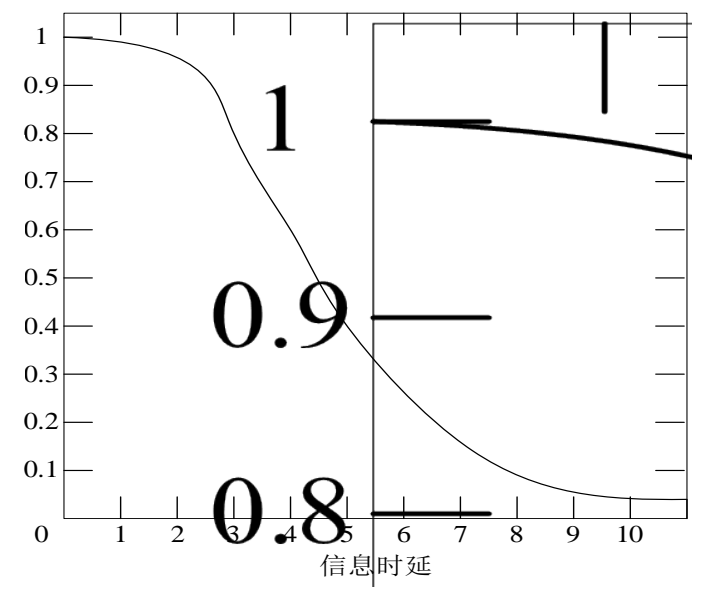

Figure7 Influence of information time delay for the probability of reporting battlefield situation

When intelligence center for an intelligence packet processing time is 1 , we can successfully obtain valid information; when intelligence center for an intelligence packet processing time is 5, we can command troops carry through tactical operations, however, because of inaccurate intelligence, cannot achieve effective situation; when intelligence center for an intelligence packet processing time is 10 , owing to the long processing time, there is no time for us to reporting or causing packet missing.

\section{Conclusion}

We combine IDEF method Petri nets to establish intelligence process simulation model which can effectively reflect the intelligence information activities of command and communication system of digitization Armored Division have the characteristics of concurrent and non-linear. By running the model, we can effectively analyze the rationality and feasibility of the work division of the headquarters, and discover the problems in process that make the command activities more orderly, and maintain efficient work of the every component in the command structure and maintain a certain ratio and optimum relationship of each component.

\section{References}

[1] Liu Leibo, Liu Huimin, Huang Ming. Headquarters construction in informationization conditions [M].Beijing: National Defense University Press, 2005(16).

[2] ZHU Jian, Dai Jianwei, Wang Wenge. Command Modeling and Simulation of the status and development of information systems [J].Ordnance Industry Automation 2009, 28 (5).

[3] Shang Wenli, Wang Chengen. System modeling method based on IDEF and UML [J]. Computer Integrated Manufacturing Systems 2004, 10 (3).

[4] Chen Yuliu .IDEF modeling analysis and design methods [M].Beijing: Tsinghua University Press, 1995.

[5] Zhang Pengcheng, Li Renhou. Thick fuzzy colored Petri nets and its applications in workflow modeling [J].Computer-Aided Design and Computer Graphics, 2002,14 (8): 713-716.

[6] Cao Huagong, Yang Manhong. defined object Petri net-based workflow process definition based on object Petri nets[J].Computer-Aided Design and Computer Graphics, 2001,13 (1): 13-18. 